\title{
Water deficit on the physiological, morphoagronomic, and technological traits of carioca common bean genotypes
}

Gabriel de Moraes Cunha Gonçalves ${ }^{1 *}\left(\mathbb{0}\right.$, João Guilherme Ribeiro Gonçalves ${ }^{1}{ }^{\circledR}$, Jean Fausto de Carvalho Paulino ${ }^{2}$, Caléo Panhoca de Almeida $^{2}$, Sérgio Augusto Morais Carbonell ${ }^{10}$, Alisson Fernando Chiorato ${ }^{10}$

Instituto Agronômico de Campinas/Centro de Grãos e Fibras, Av. Dr. Theodureto de Almeida Camargo, $1500-$ 13075-630 - Campinas, SP - Brasil.

2Instituto Agronômico de Campinas/Centro de Recursos Genéticos, Av. Dr. Theodureto de Almeida Camargo, 1500 13075-630 - Campinas, SP - Brasil.

*Corresponding author <gabriel_demoraes@hotmail.com>

Edited by: Leonardo Oliveira Medici

Received January 25, 2021

Accepted March 29, 2021
ABSTRACT: Water deficit can alter the morphological, agronomic, physiological, and technological traits of the common bean plant, affecting bean grain yield. In addition to yield aspects, the grain post-harvest quality is a decisive factor for the adoption of a new cultivar. Thus, this study evaluated the effect of water deficit on the physiological, morphoagronomic, and technological traits of common bean. The experiment was carried out at in a greenhouse in a randomized block design with a $30 \times 2$ factorial arrangement, consisting of 30 carioca common bean genotypes and two water regimes (with and without water stress), with three replications. The water deficit affected most morphological, agronomic, and physiological traits; however, it was not significant on the darkening of the seed coat. Cultivars IAC 1849 Polaco, ANFC 5, ANFc 9, BRSMG Madrepérola, IAC Carioca Aruã, TAA Dama, and Branquinho exhibited high yield potential under water stress treatment as well as slow seed coat darkening during nine months of storage. The selection of bean genotypes with slow seed coat darkening could be performed at 30 days of storage.

Keywords: Phaseolus vulgaris L., plant breeding, abiotic stress, seed coat darkening, postharvest quality

\section{Introduction}

Common bean (Phaseolus vulgaris L.) is a stable food and has high socioeconomic importance in Brazil. The average consumption is $17 \mathrm{~kg}$ person ${ }^{-1} \mathrm{yr}^{-1}$, with frequency of consumption from three to seven times a week (Ribeiro et al., 2019a). Carioca (cream-colored seed coat with brown streaks) common bean is most preferred by Brazilian consumers, followed by black bean and other types (Faria et al., 2008).

Breeding programs for common bean are important to increase crop yield and genetic gains in seed yield range from 0.5 to $6.7 \%$ per year (Chiorato et al., 2010; Barili et al., 2016; Zeffa et al., 2020). Nevertheless, yield of cultivars is still below the potential of species (Beebe et al., 2013), especially because of biotic and abiotic factors that affect plant growth and development (Fahad et al., 2017). Drought is an abiotic factor that can reduce seed yield from 10 to $100 \%$ (Dipp et al., 2017; Egu, 2016; Lanna et al., 2016; Rao, 2014).

Around $60 \%$ of bean crops worldwide are affected by terminal or intermittent drought stress. In Brazil, terminal drought occurs more frequently in the northeastern region, while intermittent drought can affect all bean producing regions (Beebe et al., 2013; Oya et al., 2004). The effects of water deficit on common bean plants have been extensively studied on the physiological, morphological, and agronomic levels (Arruda et al., 2018; Androcioli et al., 2020; Gonçalves et al., 2019; Kazai et al., 2019; Ribeiro et al., 2019b; Smith et al., 2019).

Furthermore, the technological quality of bean seeds, such as cooking time and seed coat color, is a decisive factor for the adoption of a new cultivar. The speed or rate at which the bean seed coat darkens is one of the traits with most relevance for common bean growers. Abiotic stresses affect not only seed yield, but also seed quality, hardening and darkening the seed, in addition to reducing seed size and weight. Thus, the breeding of cultivars that adapt to unfavorable environments, maintaining yield and seed quality under, has become an essential strategy for food security (Mutava et al., 2015).

This study evaluated 30 genotypes of carioca seed coat common bean on the effect of water deficit on physiological, morphoagronomic, and technological traits.

\section{Materials and Methods}

\section{Plant material and experimental design for physiological and morphoagronomic traits}

The experiment was carried out at in a greenhouse in Campinas, São Paulo, Brazil $\left(22^{\circ} 54^{\prime} \mathrm{S}, 47^{\circ} 03^{\prime} \mathrm{W}\right.$, altitude of $854 \mathrm{~m}$ ) using a randomized block design in a $30 \times$ 2 factorial arrangement composed of 30 genotypes of common bean evaluated under two water regimes (WR) (irrigated and water deficit), with three replications. The following genotypes were used: IAC Carioca Aruã, IAC Carioca Eté, IAC Carioca Tybatã, IAC Alvorada, IAC Formoso, IAC Imperador, IAC Sintonia, IAC Milênio, IAC 1850, IAC 1849 Polaco, Gen TS 3-3, and Gen TS 4-8, from the breeding program of the Agronomic Institute (IAC); Branquinho, a landrace of common bean; Pérola, BRS Pontal, BRS Horizonte, BRS Requinte, BRS Estilo, 
BRS FC 402, and BRSMG Madrepérola, from the Brazilian Agricultural Research Corporation (Embrapa); IPR Tangará, IPR Quero-Quero, IPR Sabiá, IPR Campos Gerais, IPR Curió, and IPR Celeiro, from the Agronomic Institute of Paraná (IAPAR); TAA Dama, and TAA Gol, from the Agropecuária Terras Altas; and ANFc 9, and ANFc 5, from the Agronorte Pesquisa e Sementes.

These genotypes represent most cultivars of the common bean seed market in the last 20 years, as well as two lines developed by the IAC bean breeding program in the final phase of evaluation for possible market release (Gen TS 3-3, and Gen TS 4-8).

After pre-germination of seeds in a laboratory oven, three seedlings were transferred to $10 \mathrm{dm}^{-3}$ capacity plastic pots filled with a mixture of soil and cured cattle manure at the ratio $3: 1$. The plants were thinned upon reaching the V3 phenological stage (first fully expanded three leaflet leaves), leaving two plants per pot.

The treatments were irrigated twice a day for 1 min, distributing $140 \mathrm{~mL}$ of water per pot each time through feeder lines and a spaghetti tube for each pot. The matric potential of the soil was kept at around -30 centibar / $\mathrm{kPa}$, monitored daily by moisture sensors set up randomly in the pots (Gonçalves et al., 2019).

The water deficit applied was intermittent, beginning at R5 (appearance of first flower buds) phenological stage. Short periods of water deficit were applied, alternating with irrigation periods, monitored by soil moisture sensors in which readings near -199 centibar / $\mathrm{kPa}$ indicated water scarcity in the soil. The first water deficit cycle lasted five days, when plants exhibited severe wilting due to water shortage in the soil. To avoid permanent wilting, irrigation was resumed for two days and, immediately after, two more of the same water deficit cycles were applied. In the third water deficit cycle, physiological and morphological evaluations were performed and then irrigation was reestablished until the end of the crop cycle (Gonçalves et al., 2019).

At the time of maximum stress, the following evaluations were performed: relative water content (RWC) (Jamaux et al., 1997), relative chlorophyll content (RCC) by SPAD-502Plus in lower leaves of the plant, and leaf temperature (LT) through an infrared thermometer, with the reading taken at $50 \mathrm{~cm}$ from the leaf surface at a $45^{\circ}$ angle.

The following morphological traits were evaluated: leaf area (LA), through an area meter; total shoot dry matter (TDM), where the plants used for evaluation of leaf area were kept in a forced air circulation laboratory oven at $60{ }^{\circ} \mathrm{C}$ until reaching constant weight; number of nodes per plant $(\mathrm{NN})$; plant height $(\mathrm{PH})$ in centimeters; and root collar diameter (RCD), using a digital caliper rule.

After the plants were collected in the R9 stage (physiological maturity), the following traits were evaluated: number of seeds per plant (NSP), number of pods per plant (NPP), number of empty locules (NEL), number of unviable pods (NUP), seed yield (SY) (g per plant, number of days to flowering (NDF), and number of days to maturity (NDM).

\section{Technological traits evaluated}

A seed sample was removed from each experimental plot to evaluate seed coat darkening during the storage period. The seed was placed in plastic bags $(8.5 \times 12 \mathrm{~cm})$, appropriately sealed, and randomly stored on shelves with artificial lighting. The bags were repositioned every two weeks to simulate market conditions and offer uniform lighting to the seed. Evaluations were made at monthly intervals during the storage time, namely, at 0 (harvest date), 30, 60, 90, 120, 150, 180, 210, 240, and 270 days, in a randomized block experimental design in a $30 \times 2 \times 10$ factorial arrangement, with three replications. Treatments consisted of the combination of 30 common bean genotypes (G), two water regimes (WR) (irrigated and water deficit), and 10 seed storage times (ST).

Seed coat darkening was evaluated from the value of L (lightness), ranging from 0 (black) to 100 (white). Seed coat darkening was read using a colorimeter. After 270 days of storage, the samples were evaluated regarding mean cooking time $(\mathrm{CT})$ in minutes with the assistance of a Mattson cooker, following the protocol established by Proctor and Watts (1987).

\section{Statistical analysis}

The analysis of variance was performed for the physiological, morphological, and agronomic traits, and the mean values were clustered by the Scott-Knott test at $5 \%$ probability. The analysis of variance was performed on the seed coat darkening data and the effect of storage time was decomposed through the first-degree linear regression analysis (Arns et al., 2018). To check for association among the traits evaluated, the Pearson correlation analysis was performed. The analyses were conducted with the assistance of the Genes software (Cruz, 2013) and the software R (R Core Team, version 4.0.3).

The water stress intensity index (SII) (Fischer and Maurer, 1978) was calculated by the following formula:

$S I I=\frac{\bar{x} d \cdot h}{\bar{x} i}$

where: $\bar{x} d . h$ is the mean production of all the genotypes under water deficit, and $\bar{x} i$ is the mean production of all the genotypes under irrigated conditions.

The drought tolerance index (DTI) (Darkwa et al., 2016) was calculated, for each genotype, by the following formula:

$D T I=\frac{Y d s \times Y n s}{(X d s)^{2}}$ 
where: Yds and Yns are the mean yield values per plant of a given genotype in the drought stress and non-stress conditions, respectively, and $X d s$ is the mean of all genotypes under the drought stress.

\section{Results}

\section{Effect of the water treatment on the physiological and morphoagronomic traits}

The results of analysis of variance showed a significant effect $(p \leq 0.05)$ for genotypes for the traits $\mathrm{NN}, \mathrm{PH}$, TDM, NPP, NDF, NDM, and CT, revealing variability among the genotypes studied. The effects of water regimes were significant for the traits RWC, LT, LA, PH,
TDM, NSP, NPP, SY, NDM, and CT ( $p \leq 0.05)$. Water deficit reduced the phenotypic mean of most traits evaluated, except for leaf temperature and cooking time. The traits NPP, NEL, SY, and CT showed significant effects for the genotype $\times$ water regimes interaction $(p$ $\leq 0.05)$. The coefficients of variation $(\mathrm{CV})$ ranged from 4.6 (NDM) to $36.9 \%$ (PH) (Tables 1 and 2).

The water deficit applied generated a stress intensity index of $45.4 \%$, leading to a significant reduction of $2.4 \%$ (NDM), $19.5 \%$ (RWC), $24.2 \%$ (NPP), $37.2 \%(\mathrm{PH}), 38.5 \%(\mathrm{LA}), 39.5 \%$ (NSP), $44.0 \%$ (TDM), and $45.5 \%$ (SY), compared to the irrigated treatment. In contrast, leaf temperature increased by $35.4 \%$ and cooking time by $38.5 \%$ in genotypes under water restriction.

Table 1 - Mean values of relative water content (RWC), leaf temperature (LT), leaf area (LA), plant height (PH), and total dry matter (TDM) of 30 common bean genotypes evaluated under two water treatments (irrigated and water deficit).

\begin{tabular}{|c|c|c|c|c|c|c|c|c|c|c|}
\hline \multirow{2}{*}{ ID } & \multicolumn{2}{|c|}{ RWC } & \multicolumn{2}{|c|}{ LT } & \multicolumn{2}{|c|}{ LA } & \multicolumn{2}{|c|}{$\mathrm{PH}$} & \multicolumn{2}{|c|}{ TDM } \\
\hline & Irrigated & Deficit & Irrigated & Deficit & Irrigated & Deficit & Irrigated & Deficit & Irrigated & Deficit \\
\hline & \multicolumn{2}{|c|}{$\%$} & \multicolumn{2}{|c|}{${ }^{\circ} \mathrm{C}$} & \multicolumn{2}{|c|}{$\mathrm{cm}^{2}$} & \multicolumn{2}{|c|}{$\mathrm{cm}$} & \multicolumn{2}{|c|}{$\mathrm{g}$} \\
\hline 1 & $78.0 \mathrm{Aa}$ & $69.0 \mathrm{Aa}$ & $23.4 \mathrm{Ba}$ & $31.2 \mathrm{Aa}$ & $2863.3 \mathrm{Aa}$ & $1573.3 \mathrm{Aa}$ & $70.3 \mathrm{Ab}$ & $38.3 \mathrm{Ab}$ & $10.9 \mathrm{Aa}$ & 4.2 Ba \\
\hline 2 & 85.0 Aa & $61.0 \mathrm{Ba}$ & $22.8 \mathrm{Ba}$ & 33.0 Аa & $3066.6 \mathrm{Aa}$ & 2506.6 Aa & $59.6 \mathrm{Ab}$ & $58.0 \mathrm{Ab}$ & $10.6 \mathrm{Aa}$ & $6.6 \mathrm{Aa}$ \\
\hline 3 & $76.0 \mathrm{Aa}$ & $60.0 \mathrm{Aa}$ & $23.4 \mathrm{Ba}$ & $31.3 \mathrm{Aa}$ & $3600.0 \mathrm{Aa}$ & $2183.3 \mathrm{Aa}$ & $126.0 \mathrm{Aa}$ & $46.0 \mathrm{Bb}$ & $10.7 \mathrm{Aa}$ & $6.1 \mathrm{Aa}$ \\
\hline 4 & $84.0 \mathrm{Aa}$ & $63.0 \mathrm{Ba}$ & $22.0 \mathrm{Ba}$ & $30.0 \mathrm{Aa}$ & 4393.3 Aa & $2186.6 \mathrm{Ba}$ & $108.6 \mathrm{Ab}$ & $100.6 \mathrm{Aa}$ & $13.4 \mathrm{Aa}$ & $6.2 \mathrm{Ba}$ \\
\hline 5 & $82.0 \mathrm{Aa}$ & $67.0 \mathrm{Aa}$ & $22.2 \mathrm{Ba}$ & $31.3 \mathrm{Aa}$ & 4076.6 Aa & $2150.0 \mathrm{Ba}$ & $73.6 \mathrm{Ab}$ & $37.0 \mathrm{Ab}$ & $10.8 \mathrm{Aa}$ & $5.8 \mathrm{Ba}$ \\
\hline 6 & $80.0 \mathrm{Aa}$ & $68.0 \mathrm{Aa}$ & $24.1 \mathrm{Ba}$ & $32.8 \mathrm{Aa}$ & $3560.0 \mathrm{Aa}$ & $2296.6 \mathrm{Aa}$ & $60.0 \mathrm{Ab}$ & $55.6 \mathrm{Ab}$ & $10.9 \mathrm{Aa}$ & $6.2 \mathrm{Aa}$ \\
\hline 7 & $85.0 \mathrm{Aa}$ & $70.0 \mathrm{Aa}$ & 23.7 Ba & $30.3 \mathrm{Aa}$ & 3493.3 Aa & $1780.0 \mathrm{Ba}$ & 103.3 Ab & $50.0 \mathrm{Ab}$ & $11.4 \mathrm{Aa}$ & $5.0 \mathrm{Ba}$ \\
\hline 8 & 76.0 Aa & $67.0 \mathrm{Aa}$ & $24.3 \mathrm{Ba}$ & $32.4 \mathrm{Aa}$ & $3760.0 \mathrm{Aa}$ & $2200.0 \mathrm{Ba}$ & 124.6 Aa & $47.0 \mathrm{Bb}$ & $11.3 \mathrm{Aa}$ & $6.4 \mathrm{Ba}$ \\
\hline 9 & 85.0 Aa & $64.0 \mathrm{Ba}$ & 23.2 Ba & $29.3 \mathrm{Aa}$ & $3566.0 \mathrm{Aa}$ & $2106.6 \mathrm{Aa}$ & $102.3 \mathrm{Ab}$ & $56.0 \mathrm{Ab}$ & $9.9 \mathrm{Aa}$ & $6.1 \mathrm{Aa}$ \\
\hline 10 & $78.0 \mathrm{Aa}$ & $66.0 \mathrm{Aa}$ & $25.1 \mathrm{Ba}$ & $30.9 \mathrm{Aa}$ & $2433.3 \mathrm{Aa}$ & $1560.0 \mathrm{Aa}$ & $44.3 \mathrm{Ab}$ & $43.3 \mathrm{Ab}$ & $6.5 \mathrm{Aa}$ & $4.2 \mathrm{Aa}$ \\
\hline 11 & $76.9 \mathrm{Aa}$ & $62.0 \mathrm{Aa}$ & $24.0 \mathrm{Ba}$ & $30.1 \mathrm{Aa}$ & $3060.0 \mathrm{Aa}$ & $1623.3 \mathrm{Aa}$ & $66.0 \mathrm{Ab}$ & $34.0 \mathrm{Ab}$ & $11.1 \mathrm{Aa}$ & $3.9 \mathrm{Ba}$ \\
\hline 12 & $84.0 \mathrm{Aa}$ & $55.0 \mathrm{Ba}$ & $24.0 \mathrm{Ba}$ & 31.9 Аа & 3196.6 Аa & $2430.0 \mathrm{Aa}$ & $94.3 \mathrm{Ab}$ & 87.6 Аa & $8.8 \mathrm{Aa}$ & $7.4 \mathrm{Aa}$ \\
\hline 13 & $87.0 \mathrm{Aa}$ & $72.0 \mathrm{Aa}$ & $23.0 \mathrm{Ba}$ & $29.9 \mathrm{Aa}$ & 3323.3 Aa & $1570.0 \mathrm{Ba}$ & 107.3 Ab & $46.0 \mathrm{Ab}$ & $9.5 \mathrm{Aa}$ & $3.8 \mathrm{Ba}$ \\
\hline 14 & $80.0 \mathrm{Aa}$ & 73.0 Aa & $22.4 \mathrm{Ba}$ & $31.6 \mathrm{Aa}$ & 2993.3 Аa & 1896.6 Aa & 184.6 Аa & $117.0 \mathrm{Ba}$ & $12.0 \mathrm{Aa}$ & $6.4 \mathrm{Ba}$ \\
\hline 15 & $86.0 \mathrm{Aa}$ & $66.0 \mathrm{Ba}$ & $23.1 \mathrm{Ba}$ & $31.5 \mathrm{Aa}$ & $3156.6 \mathrm{Aa}$ & $1913.3 \mathrm{Aa}$ & 137.6 Aa & $56.6 \mathrm{Bb}$ & $10.6 \mathrm{Aa}$ & $5.4 \mathrm{Ba}$ \\
\hline 16 & $92.0 \mathrm{Aa}$ & $70.0 \mathrm{Ba}$ & $21.9 \mathrm{Ba}$ & $30.6 \mathrm{Aa}$ & $2730.0 \mathrm{Aa}$ & $1710.0 \mathrm{Aa}$ & 78.6 Ab & $38.6 \mathrm{Ab}$ & $8.9 \mathrm{Aa}$ & $4.7 \mathrm{Aa}$ \\
\hline 17 & $78.0 \mathrm{Aa}$ & $72.0 \mathrm{Aa}$ & 23.4 Ba & 32.7 Aa & 3023.3 Аa & $1973.3 \mathrm{Aa}$ & $102.3 \mathrm{Ab}$ & $92.3 \mathrm{Aa}$ & $9.7 \mathrm{Aa}$ & $5.7 \mathrm{Aa}$ \\
\hline 18 & 86.0 Aa & $68.0 \mathrm{Ba}$ & 23.4 Ba & $30.0 \mathrm{Aa}$ & 4296.6 Аa & $2010.0 \mathrm{Ba}$ & 104.0 Ab & $46.3 \mathrm{Ab}$ & 13.8 Aa & $5.9 \mathrm{Ba}$ \\
\hline 19 & $89.0 \mathrm{Aa}$ & $65.0 \mathrm{Ba}$ & $21.7 \mathrm{Ba}$ & $30.9 \mathrm{Aa}$ & $3266.6 \mathrm{Aa}$ & $2316.6 \mathrm{Aa}$ & 153.3 Аа & $87.3 \mathrm{Ba}$ & $8.9 \mathrm{Aa}$ & $7.5 \mathrm{Aa}$ \\
\hline 20 & $85.0 \mathrm{Aa}$ & $66.0 \mathrm{Ba}$ & $22.6 \mathrm{Ba}$ & 31.4 Aa & $3006.6 \mathrm{Aa}$ & $1796.6 \mathrm{Aa}$ & $114.3 \mathrm{Aa}$ & $76.3 \mathrm{Aa}$ & $6.9 \mathrm{Aa}$ & $5.4 \mathrm{Aa}$ \\
\hline 21 & $87.0 \mathrm{Aa}$ & $67.0 \mathrm{Ba}$ & $22.4 \mathrm{Ba}$ & $32.5 \mathrm{Aa}$ & $3473.3 \mathrm{Aa}$ & $2126.6 \mathrm{Aa}$ & 133.3 Аа & $77.3 \mathrm{Aa}$ & $12.7 \mathrm{Aa}$ & $7.1 \mathrm{Ba}$ \\
\hline 22 & 75.0 Aa & $56.0 \mathrm{Ba}$ & $24.6 \mathrm{Ba}$ & 33.2 Aa & $2910.0 \mathrm{Aa}$ & $2250.0 \mathrm{Aa}$ & $97.0 \mathrm{Ab}$ & $73.3 \mathrm{Aa}$ & $9.5 \mathrm{Aa}$ & 7.4 Aa \\
\hline 23 & $80.0 \mathrm{Aa}$ & $60.0 \mathrm{Ba}$ & $22.2 \mathrm{Ba}$ & $31.7 \mathrm{Aa}$ & 3763.3 Аа & $2510.0 \mathrm{Aa}$ & 161.0 Aa & $71.3 \mathrm{Ba}$ & $12.2 \mathrm{Aa}$ & $6.3 \mathrm{Ba}$ \\
\hline 24 & 88.0 Aa & $62.0 \mathrm{Ba}$ & $22.7 \mathrm{Ba}$ & 32.0 Aa & 2753.3 Aa & $2100.0 \mathrm{Aa}$ & 122.6 Aa & $60.3 \mathrm{Ab}$ & $7.4 \mathrm{Aa}$ & $5.8 \mathrm{Aa}$ \\
\hline 25 & $85.0 \mathrm{Aa}$ & $65.0 \mathrm{Ba}$ & $22.6 \mathrm{Ba}$ & $31.6 \mathrm{Aa}$ & $2706.6 \mathrm{Aa}$ & $1866.6 \mathrm{Aa}$ & $65.3 \mathrm{Ab}$ & $82.0 \mathrm{Aa}$ & $9.3 \mathrm{Aa}$ & $4.9 \mathrm{Aa}$ \\
\hline 26 & $82.0 \mathrm{Aa}$ & $72.0 \mathrm{Aa}$ & 23.4 Ba & 31.1 Aa & $2840.0 \mathrm{Aa}$ & $1686.6 \mathrm{Aa}$ & $75.0 \mathrm{Ab}$ & $33.3 \mathrm{Ab}$ & $9.4 \mathrm{Aa}$ & 3.7 Ba \\
\hline 27 & $78.0 \mathrm{Aa}$ & $67.0 \mathrm{Aa}$ & $22.5 \mathrm{Ba}$ & 33.2 Aa & $3266.6 \mathrm{Aa}$ & $2326.6 \mathrm{Aa}$ & $129.6 \mathrm{Aa}$ & $140.6 \mathrm{Aa}$ & $12.0 \mathrm{Aa}$ & $8.0 \mathrm{Aa}$ \\
\hline 28 & $80.0 \mathrm{Aa}$ & $67.0 \mathrm{Aa}$ & $24.5 \mathrm{Ba}$ & $29.9 \mathrm{Aa}$ & 3106.6 Aa & 1943.3 Aa & $55.3 \mathrm{Ab}$ & $56.0 \mathrm{Ab}$ & $9.4 \mathrm{Aa}$ & $4.8 \mathrm{Aa}$ \\
\hline 29 & $80.0 \mathrm{Aa}$ & $70.0 \mathrm{Aa}$ & $23.1 \mathrm{Ba}$ & $31.8 \mathrm{Aa}$ & $2816.6 \mathrm{Aa}$ & 1696.6 Aa & $177.6 \mathrm{Aa}$ & $82.0 \mathrm{Ba}$ & $10.8 \mathrm{Aa}$ & $5.8 \mathrm{Ba}$ \\
\hline 30 & $73.0 \mathrm{Aa}$ & $57.0 \mathrm{Aa}$ & 23.4 Ba & $31.9 \mathrm{Aa}$ & $3483.3 \mathrm{Aa}$ & $1946.6 \mathrm{Ba}$ & 107.6 Ab & $80.3 \mathrm{Aa}$ & $11.2 \mathrm{Aa}$ & 6.4 Ba \\
\hline Mean & $82.0 \mathrm{~A}$ & $66.0 \mathrm{~B}$ & $23.2 \mathrm{~B}$ & $31.4 \mathrm{~A}$ & $3266.2 \mathrm{~A}$ & 2007.8 B & $104.6 \mathrm{~A}$ & $65.7 \mathrm{~B}$ & $10.4 \mathrm{~A}$ & $5.8 \mathrm{~B}$ \\
\hline CV (\%) & \multicolumn{2}{|c|}{10.8} & \multicolumn{2}{|c|}{8.1} & \multicolumn{2}{|c|}{17.0} & \multicolumn{2}{|c|}{36.9} & \multicolumn{2}{|c|}{28} \\
\hline
\end{tabular}

Mean values followed by the same uppercase letters in the row and lowercase letters in the column do not differ from each other by the Scott-Knott test ( $p \leq 0.05$ ) for water treatment and genotypes respectively. ID (identification): Genotypes: 1 - IAC Carioca Aruã, 2 - IAC Carioca Eté, 3 - IAC Carioca Tybatã, 4 - IAC Alvorada, 5 - IAC Formoso, 6 - IAC Imperador, 7 - IAC Sintonia, 8 - IAC Milênio, 9 - IAC 1850, 10 - IAC 1849 Polaco, 11 - Gen TS 3-3, 12 - Gen TS 4-8, 13 - Branquinho, 14 - Pérola, 15 - BRS Pontal, 16 - BRS Horizonte, 17 - BRS Requinte, 18 - BRS Estilo, 19 - BRS FC 402,20 - BRSMG Madrepérola, 21 - IPR Tangará, 22 - IPR Quero-Quero, 23 - IPR Sabiá, 24 - IPR Campos Gerais, 25 - IPR Curió, 26 - IPR Celeiro, 27 - TAA Dama, 28 - TAA Gol, 29 - ANFc 9, 30 - ANFc 5. 
Table 2 - Mean values of number of seeds per plant (NSP), number of pods per plant (NPP), seed yield (SY), number of days to maturity (NDM), and cooking time (CT) of 30 common bean genotypes evaluated under two water treatments (irrigated and water deficit).

\begin{tabular}{|c|c|c|c|c|c|c|c|c|c|c|}
\hline \multirow{2}{*}{ ID } & \multicolumn{2}{|c|}{ NSP } & \multicolumn{2}{|c|}{ NPP } & \multicolumn{2}{|c|}{ SY } & \multicolumn{2}{|c|}{ NDM } & \multicolumn{2}{|c|}{ CT } \\
\hline & Irrigated & Deficit & Irrigated & Deficit & Irrigated & Deficit & Irrigated & Deficit & Irrigated & Deficit \\
\hline & \multicolumn{4}{|c|}{$\longrightarrow$ unit } & \multicolumn{2}{|c|}{ g per plant ___ } & \multicolumn{2}{|c|}{ days } & \multicolumn{2}{|c|}{$-\min \longrightarrow$} \\
\hline 1 & $37.6 \mathrm{Aa}$ & $33.67 \mathrm{Aa}$ & $14.0 \mathrm{Aa}$ & $7.6 \mathrm{Bc}$ & $12.4 \mathrm{Aa}$ & $10.1 \mathrm{Aa}$ & $78.0 \mathrm{Aa}$ & $79.0 \mathrm{Aa}$ & $25.7 \mathrm{Ab}$ & $30.7 \mathrm{Ad}$ \\
\hline 2 & 48.3 Aa & $23.6 \mathrm{Ba}$ & $14.3 \mathrm{Aa}$ & $4.0 \mathrm{Bc}$ & 14.0 Aa & 3.3 Ba & $76.3 \mathrm{Aa}$ & $80.3 \mathrm{Aa}$ & $41.6 \mathrm{Ba}$ & $72.9 \mathrm{Ab}$ \\
\hline 3 & $55.3 \mathrm{Aa}$ & $27.0 \mathrm{Ba}$ & $12.0 \mathrm{Aa}$ & 7.6 Ac & 12.3 $\mathrm{Aa}$ & $6.0 \mathrm{Aa}$ & $76.0 \mathrm{Aa}$ & $80.0 \mathrm{Aa}$ & $49.8 \mathrm{Ba}$ & $68.2 \mathrm{Ab}$ \\
\hline 4 & 39.6 Aa & $16.6 \mathrm{Ba}$ & $10.6 \mathrm{Aa}$ & 4.6 Bc & 13.4 Aa & $5.1 \mathrm{Ba}$ & $77.0 \mathrm{Aa}$ & 73.0 Ab & $37.9 \mathrm{Ba}$ & $57.0 \mathrm{Ac}$ \\
\hline 5 & $50.6 \mathrm{Aa}$ & $31.6 \mathrm{Aa}$ & $9.6 \mathrm{Aa}$ & $8.3 \mathrm{Ac}$ & $14.1 \mathrm{Aa}$ & $7.4 \mathrm{Aa}$ & $76.3 \mathrm{Aa}$ & $71.0 \mathrm{Ab}$ & $47.8 \mathrm{Aa}$ & $49.4 \mathrm{Ac}$ \\
\hline 6 & $34.0 \mathrm{Aa}$ & $31.6 \mathrm{Aa}$ & $9.0 \mathrm{Aa}$ & $12.3 \mathrm{Ab}$ & $10.3 \mathrm{Aa}$ & $8.1 \mathrm{Aa}$ & $76.0 \mathrm{Aa}$ & $74.0 \mathrm{Ab}$ & $42.2 \mathrm{Aa}$ & $47.2 \mathrm{Ac}$ \\
\hline 7 & $39.6 \mathrm{Aa}$ & $21.3 \mathrm{Aa}$ & $11.6 \mathrm{Aa}$ & $7.0 \mathrm{Bc}$ & 12.6 Aa & $6.8 \mathrm{Aa}$ & $76.0 \mathrm{Aa}$ & $74.6 \mathrm{Ab}$ & $41.7 \mathrm{Aa}$ & $45.3 \mathrm{Ac}$ \\
\hline 8 & 37.0 Aa & $20.0 \mathrm{Aa}$ & $9.6 \mathrm{Aa}$ & $6.3 \mathrm{Ac}$ & $11.1 \mathrm{Aa}$ & 4.2 Ba & $79.3 \mathrm{Aa}$ & 81.3 Aa & $34.5 \mathrm{Bb}$ & $50.5 \mathrm{Ac}$ \\
\hline 9 & $65.3 \mathrm{Aa}$ & $37.6 \mathrm{Aa}$ & $13.0 \mathrm{Ba}$ & $24.6 \mathrm{Aa}$ & $17.5 \mathrm{Aa}$ & $10.7 \mathrm{Ba}$ & $75.0 \mathrm{Aa}$ & $74.0 \mathrm{Ab}$ & 47.4 Ba & $64.9 \mathrm{Ab}$ \\
\hline 10 & 48.3 Aa & $35.6 \mathrm{Aa}$ & 13.6 Aa & $11.0 \mathrm{Ab}$ & $11.3 \mathrm{Aa}$ & $9.5 \mathrm{Aa}$ & $72.6 \mathrm{Aa}$ & $71.6 \mathrm{Ab}$ & $55.2 \mathrm{Aa}$ & $53.3 \mathrm{Ac}$ \\
\hline 11 & $36.0 \mathrm{Aa}$ & $17.6 \mathrm{Aa}$ & $9.3 \mathrm{Aa}$ & $7.0 \mathrm{Ac}$ & $10.7 \mathrm{Aa}$ & $5.3 \mathrm{Aa}$ & $75.0 \mathrm{Aa}$ & $70.3 \mathrm{Ab}$ & $35.3 \mathrm{Bb}$ & $53.9 \mathrm{Ac}$ \\
\hline 12 & 39.6 Aa & $21.6 \mathrm{Aa}$ & $10.3 \mathrm{Aa}$ & $5.3 \mathrm{Bc}$ & $13.8 \mathrm{Aa}$ & $4.7 \mathrm{Ba}$ & $76.0 \mathrm{Aa}$ & 76.6 Aa & $41.6 \mathrm{Ba}$ & $56.0 \mathrm{Ac}$ \\
\hline 13 & 43.6 Aa & $29.6 \mathrm{Aa}$ & $8.6 \mathrm{Aa}$ & 7.6 Ac & $10.1 \mathrm{Aa}$ & $6.2 \mathrm{Aa}$ & $77.0 \mathrm{Aa}$ & $74.0 \mathrm{Ab}$ & $41.2 \mathrm{Ba}$ & $55.2 \mathrm{Ac}$ \\
\hline 14 & 41.6 Aa & $23.3 \mathrm{Aa}$ & $11.3 \mathrm{Aa}$ & $10.6 \mathrm{Ab}$ & $12.4 \mathrm{Aa}$ & $5.6 \mathrm{Ba}$ & $81.0 \mathrm{Aa}$ & 79.6 Aa & $47.6 \mathrm{Ba}$ & $72.8 \mathrm{Ab}$ \\
\hline 15 & 45.3 Aa & $31.6 \mathrm{Aa}$ & $8.6 \mathrm{Aa}$ & $8.0 \mathrm{Ac}$ & $10.6 \mathrm{Aa}$ & $8.0 \mathrm{Aa}$ & $75.0 \mathrm{Aa}$ & $74.3 \mathrm{Ab}$ & $42.7 \mathrm{Ba}$ & $61.4 \mathrm{Ab}$ \\
\hline 16 & 44.0 Aa & $24.6 \mathrm{Aa}$ & $9.6 \mathrm{Aa}$ & $6.0 \mathrm{Ac}$ & $12.2 \mathrm{Aa}$ & $6.5 \mathrm{Aa}$ & $75.0 \mathrm{Aa}$ & $69.0 \mathrm{Ab}$ & $38.2 \mathrm{Aa}$ & $47.1 \mathrm{Ac}$ \\
\hline 17 & $68.3 \mathrm{Aa}$ & $25.3 \mathrm{Ba}$ & $13.0 \mathrm{Aa}$ & $7.6 \mathrm{Ac}$ & $15.0 \mathrm{Aa}$ & $6.3 \mathrm{Ba}$ & $79.0 \mathrm{Aa}$ & $76.3 \mathrm{Aa}$ & $44.3 \mathrm{Aa}$ & $53.7 \mathrm{Ac}$ \\
\hline 18 & 41.0 Aa & $25.6 \mathrm{Aa}$ & $8.3 \mathrm{Aa}$ & $5.3 \mathrm{Ac}$ & $12.9 \mathrm{Aa}$ & $7.1 \mathrm{Aa}$ & $77.0 \mathrm{Aa}$ & 77.6 Aa & $52.2 \mathrm{Aa}$ & $54.0 \mathrm{Ac}$ \\
\hline 19 & 42.6 Aa & $19.3 \mathrm{Ba}$ & $11.3 \mathrm{Aa}$ & $5.6 \mathrm{Bc}$ & $10.9 \mathrm{Aa}$ & $5.4 \mathrm{Aa}$ & $85.6 \mathrm{Aa}$ & 81.3 Аa & $43.5 \mathrm{Ba}$ & $61.2 \mathrm{Ab}$ \\
\hline 20 & $64.3 \mathrm{Aa}$ & $35.6 \mathrm{Ba}$ & 13.3 Aa & $7.3 \mathrm{Bc}$ & 16.1 Aa & 7.7 Ba & $79.3 \mathrm{Aa}$ & $71.3 \mathrm{Bb}$ & 42.4 Ba & $65.9 \mathrm{Ab}$ \\
\hline 21 & $35.0 \mathrm{Aa}$ & $26.6 \mathrm{Aa}$ & $7.0 \mathrm{Aa}$ & $6.0 \mathrm{Ac}$ & $11.5 \mathrm{Aa}$ & $5.0 \mathrm{Aa}$ & $78.0 \mathrm{Aa}$ & $76.3 \mathrm{Aa}$ & $38.6 \mathrm{Ba}$ & $55.8 \mathrm{Ac}$ \\
\hline 22 & 30.3 Aa & $22.6 \mathrm{Aa}$ & $11.6 \mathrm{Aa}$ & $7.3 \mathrm{Ac}$ & $9.0 \mathrm{Aa}$ & $7.0 \mathrm{Aa}$ & $80.0 \mathrm{Aa}$ & $72.6 \mathrm{Bb}$ & $40.7 \mathrm{Ba}$ & $59.4 \mathrm{Ac}$ \\
\hline 23 & $54.6 \mathrm{Aa}$ & $26.6 \mathrm{Ba}$ & $11.3 \mathrm{Aa}$ & $5.6 \mathrm{Bc}$ & $15.5 \mathrm{Aa}$ & $5.5 \mathrm{Ba}$ & $76.0 \mathrm{Aa}$ & $70.3 \mathrm{Ab}$ & $47.0 \mathrm{Ba}$ & $100.1 \mathrm{Aa}$ \\
\hline 24 & $40.0 \mathrm{Aa}$ & $20.0 \mathrm{Aa}$ & $9.0 \mathrm{Aa}$ & 7.6 Ac & $12.8 \mathrm{Aa}$ & 4.6 Ba & $75.0 \mathrm{Aa}$ & 81.0 Aa & $40.1 \mathrm{Aa}$ & $47.1 \mathrm{Ac}$ \\
\hline 25 & $39.0 \mathrm{Aa}$ & $29.6 \mathrm{Aa}$ & $10.3 \mathrm{Aa}$ & $10.6 \mathrm{Ab}$ & $12.3 \mathrm{Aa}$ & $7.6 \mathrm{Aa}$ & $73.3 \mathrm{Aa}$ & $69.0 \mathrm{Ab}$ & $30.9 \mathrm{Bb}$ & $47.9 \mathrm{Ac}$ \\
\hline 26 & 46.6 Aa & $31.3 \mathrm{Aa}$ & $11.0 \mathrm{Aa}$ & $10.6 \mathrm{Ab}$ & $12.1 \mathrm{Aa}$ & $7.5 \mathrm{Aa}$ & $76.0 \mathrm{Aa}$ & $72.3 \mathrm{Ab}$ & $42.3 \mathrm{Ba}$ & $55.0 \mathrm{Ac}$ \\
\hline 27 & $41.6 \mathrm{Aa}$ & $31.3 \mathrm{Aa}$ & $8.6 \mathrm{Aa}$ & $7.6 \mathrm{Ac}$ & $13.5 \mathrm{Aa}$ & $10.1 \mathrm{Aa}$ & $75.0 \mathrm{Aa}$ & 74.0 Ab & $34.4 \mathrm{Bb}$ & $69.6 \mathrm{Ab}$ \\
\hline 28 & $30.0 \mathrm{Aa}$ & $22.0 \mathrm{Aa}$ & $10.3 \mathrm{Aa}$ & 7.6 Ac & $10.0 \mathrm{Aa}$ & $7.1 \mathrm{Aa}$ & 73.3 Aa & $72.0 \mathrm{Ab}$ & $24.2 \mathrm{Bb}$ & $45.2 \mathrm{Ac}$ \\
\hline 29 & 42.3 Aa & $24.3 \mathrm{Aa}$ & $8.6 \mathrm{Aa}$ & $7.3 \mathrm{Ac}$ & $14.4 \mathrm{Aa}$ & $6.7 \mathrm{Ba}$ & $80.0 \mathrm{Aa}$ & $76.3 \mathrm{Aa}$ & $32.8 \mathrm{Ab}$ & $39.7 \mathrm{Ad}$ \\
\hline 30 & 36.0 Aa & $28.0 \mathrm{Aa}$ & 8.6 Aa & $6.3 \mathrm{Ac}$ & $11.1 \mathrm{Aa}$ & $7.4 \mathrm{Aa}$ & $72.3 \mathrm{Aa}$ & $70.6 \mathrm{Ab}$ & $25.2 \mathrm{Ab}$ & $35.8 \mathrm{Ad}$ \\
\hline Mean & $43.9 \mathrm{~A}$ & $26.5 \mathrm{~B}$ & $10.6 \mathrm{~A}$ & $8.0 \mathrm{~B}$ & $12.5 \mathrm{~A}$ & $6.7 \mathrm{~B}$ & $76.7 \mathrm{~A}$ & $74.8 \mathrm{~B}$ & $40.3 \mathrm{~B}$ & $55.9 \mathrm{~A}$ \\
\hline CV (\%) & \multicolumn{2}{|c|}{20.2} & \multicolumn{2}{|c|}{15.3} & \multicolumn{2}{|c|}{33.6} & \multicolumn{2}{|c|}{4.6} & \multicolumn{2}{|c|}{15.5} \\
\hline
\end{tabular}

Mean values followed by the same uppercase letters in the row and lowercase letters in the column do not differ from each other by the Scott-Knott test ( $p \leq 0.05)$ for water treatment and genotypes, respectively. ID (identification): Genotypes: 1 - IAC Carioca Aruã, 2 - IAC Carioca Eté, 3 - IAC Carioca Tybatã, 4 - IAC Alvorada, 5 - IAC Formoso, 6 - IAC Imperador, 7 - IAC Sintonia, 8 - IAC Milênio, 9 - IAC 1850, 10 - IAC 1849 Polaco, 11 - Gen TS 3-3, 12 - Gen TS 4-8, 13 - Branquinho, 14 - Pérola, 15 - BRS Pontal, 16 - BRS Horizonte, 17 - BRS Requinte, 18 - BRS Estilo, 19 - BRS FC 402, 20 - BRSMG Madrepérola, 21 - IPR Tangará, 22 - IPR Quero-Quero, 23 - IPR Sabiá, 24 - IPR Campos Gerais, 25 - IPR Curió, 26 - IPR Celeiro, 27 - TAA Dama, 28 - TAA Gol, 29 - ANFc 9, 30 - ANFc 5.

The relative water content in leaves declined for all genotypes evaluated under water deficit (Table 1). In general, a significant reduction of $19.5 \%$ do RWC was observed when water deficit was applied. Water deficit significantly increased the leaf temperature of all the genotypes evaluated, from 29.3 (IAC 1850) to $33.2{ }^{\circ} \mathrm{C}$ (TAA Dama) (Table 1).

Water deficit reduced LA by $38.5 \%$ compared to the irrigated treatment. Genotypes IAC Alvorada, BRS Estilo, IAC Formoso, IAC Milênio, IAC Sintonia, ANFc 5, and Branquinho showed significant declines in leaf areas under water deficit (Table 1). The water treatment did not change NN and RCD of the plants evaluated $(p$ $>0.05)$. Nevertheless, $\mathrm{PH}$ reduced under water deficit (Table 1).
TDM showed a mean reduction of $4.5 \mathrm{~g}$ under water deficit (Table 1). Nevertheless, the following genotypes showed a stable response, that is, they did not exhibit significant differences under the two water regimes: IAC Carioca Eté, IAC Carioca Tybatã, IAC Imperador, IAC 1850, IAC 1849 Polaco, Gen TS 4-8, BRS Horizonte, BRS Requinte, BRS FC 402, BRSMG Madrepérola, IPR Quero Quero, IPR Campos Gerais, IPR Curió, TAA Dama, and TAA Gol.

The water treatment negatively affected the NSP and the NPP. The NSP of genotypes IAC Carioca Eté, IAC Carioca Tybatã, IAC Alvorada, BRS Requinte, BRS FC 402, BRSMG Madrepérola, and IPR Sabiá declined significantly in the non-irrigated treatment (Table 2). For the NPP, significant differences $(p \leq 0.05)$ were observed 
among the genotypes under water deficit, from 4 (IAC Carioca Eté) to 24.6 pods (IAC 1850) (Table 2).

Water deficit negatively affected SY, although significant differences were not observed between the genotypes within each water treatment (Table 2). Cultivars IAC 1850, TAA Dama, and IAC Carioca Aruã were prominent, with yields per plant of $10.7 \mathrm{~g}, 10.1 \mathrm{~g}$, and $10.1 \mathrm{~g}$, respectively, in the water deficit treatment. The mean value of DTI was 1.86 (data not shown). Genotypes IAC 1850, TAA Dama, IAC Carioca Aruã, and BRSMG Madrepérola were relevant with drought tolerance index of $4.1,3.0,2.7$, and 2.7, respectively, while IAC Carioca Eté presented the lowest DTI, at 1.0.

Water deficit did not cause significant differences for the NDF variable $(p>0.05)$ however, NDM declined. The number of days to seed maturity decreased significantly for cultivars BRSMG Madrepérola and IPR Quero-Quero under water deficit (Table 2).

Water deficit led to a significant mean increase of $15.5 \mathrm{~min}$ in CT (Table 2). Under water deficit, genotypes
IAC Carioca Aruã, ANFc 5, and ANFc 9 were prominent with cooking times of $30.7 \mathrm{~min}, 35.8 \mathrm{~min}$, and 39.7 min. In contrast, cultivar IPR Sabiá showed the worst performance for CT among all genotypes evaluated, with mean seed cooking time of $100.1 \mathrm{~min}$.

\section{Effect of water treatment on seed coat darkening of common bean seed}

There were significant effects between genotypes and storage times regarding seed coat darkening ( $p \leq 0.01)$; nevertheless, differences were not significant between the water regimes for the $\mathrm{L}$ value $(p>0.05)$. Effects were also significant for the following interactions: $G \times$ $\mathrm{WR}, \mathrm{G} \times \mathrm{ST}, \mathrm{WR} \times \mathrm{ST}$, and $\mathrm{G} \times \mathrm{WR} \times \mathrm{ST}(p \leq 0.01)$.

Regardless of the water regime adopted, the firstdegree regression equations show that all genotypes evaluated had a significant reduction in the $\mathrm{L}$ value during the storage period (Table 3). Cultivars IAC 1849 Polaco, ANFc 5, ANFc 9, BRSMG Madrepérola, IAC

Table 3 - Regression equation and coefficient of determination $\left(R^{2}\right)$ for lightness $(L)$ from 30 common bean genotypes for nine months of storage under irrigated and water deficit conditions.

\begin{tabular}{|c|c|c|c|c|c|c|}
\hline \multirow{2}{*}{ Genotype } & \multicolumn{3}{|c|}{ Irrigated } & \multicolumn{3}{|c|}{ Deficit } \\
\hline & Regression & & $\mathrm{R}^{2}$ & Regression & & $\mathrm{R}^{2}$ \\
\hline & & & $\%$ & & & $\%$ \\
\hline IAC Carioca Aruã & $52.94340-0.03866 . T$ & ** & 71.0 & $52.8790-0.0385 . T$ & ** & 71.6 \\
\hline IAC Carioca Eté & $48.54847-0.06152 . T$ & * * & 82.2 & $50.05-0.05 . T$ & ** & 77.4 \\
\hline IAC Carioca Tybatã & $49.20996-0.05825 . T$ & * * & 82.1 & $50.23245-0.06236 . T$ & * * & 85.6 \\
\hline IAC Alvorada & $50.83598-0.06623 . T$ & ** & 74.2 & $50.68724-0.06114 . T$ & * * & 77.2 \\
\hline IAC Formoso & $50.15065-0.05827 . T$ & * * & 79.2 & $49.21395-0.05675 . T$ & ** & 82.7 \\
\hline IAC Imperador & $49.12605-0.06264 . T$ & ** & 74.7 & $46.80996-0.06076 . T$ & ** & 75.7 \\
\hline IAC Sintonia & $46.89756-0.05535 T$ & * * & 71.8 & $48.58136-0.05869 . T$ & * * & 74.4 \\
\hline IAC Milênio & $49.06925-0.06298 . T$ & * * & 74.8 & $50.33860-0.05778 . T$ & ** & 79.2 \\
\hline IAC 1850 & $50.60371-0.06786 . T$ & * * & 77.9 & $50.13847-0.06667 . T$ & ** & 85.7 \\
\hline IAC 1849 Polaco & $52.27076-0.03719 . T$ & * * & 81.5 & $50.40369-0.02803 . T$ & * * & 72.2 \\
\hline Gen TS 3-3 & $46.28498-0.05811 . T$ & * * & 77.3 & $44.71840-0.05258 . T$ & ** & 74.7 \\
\hline Gen TS 4-8 & $50.10476-0.06436 . T$ & * * & 77.7 & $48.14127-0.04724 . T$ & ** & 66.2 \\
\hline Branquinho & $57.16527-0.04167 . T$ & * * & 81.9 & $55.25116-0.04035 . T$ & ** & 84.4 \\
\hline Pérola & $47.16098-0.05122 . T$ & ** & 77.4 & $48.5383-0.0434 . T$ & ** & 70.9 \\
\hline BRS Pontal & $49.29276-0.05922 . T$ & * * & 81.6 & $49.44318-0.06589 . T$ & ** & 81.1 \\
\hline BRS Horizonte & $50.02945-0.06571 . T$ & * * & 75.7 & $48.81593-0.05476 . T$ & ** & 80.0 \\
\hline BRS Requinte & $50.49324-0.05457 . T$ & * * & 83.9 & $48.98084-0.05474 . T$ & ** & 86.2 \\
\hline BRS Estilo & $49.71625-0.06514 . T$ & * * & 77.3 & $51.38844-0.06233 . T$ & ** & 81.8 \\
\hline FC 402 & $48.50278-0.05924 . T$ & * * & 72.4 & $47.32458-0.05713 . T$ & * * & 75.3 \\
\hline BRSMG Madrepérola & $55.19362-0.03537 . T$ & * * & 84.4 & $53.45933-0.03594 . T$ & ** & 84.4 \\
\hline IPR Tangará & $49.40989-0.06009 . T$ & * * & 80.8 & $49.94456-0.06748 . T$ & * * & 77.0 \\
\hline IPR Quero-Quero & $45.33696-0.05089 . T$ & ** & 77.6 & $47.48049-0.05696 . T$ & ** & 81.8 \\
\hline IPR Sabiá & $50.71956-0.06843 . T$ & * * & 77.7 & $50.34584-0.06236 . T$ & ** & 76.7 \\
\hline IPR Campos Gerais & $48.9640-0.0559 . \mathrm{T}$ & * * & 75.8 & $47.74505-0.04716 . T$ & ** & 79.3 \\
\hline IPR Curió & $47.34215-0.05738 . T$ & * * & 78.4 & $47.81984-0.05867 . T$ & ** & 82.0 \\
\hline IPR Celeiro & $50.84035-0.05898 . T$ & * * & 80.9 & $50.59698-0.05845 . T$ & ** & 82.4 \\
\hline TAA Dama & $54.0878-0.0327 . T$ & * * & 83.6 & $55.13876-0.03924 . T$ & $\star *$ & 83.9 \\
\hline TAA Gol & $48.8424-0.0532 . T$ & ** & 76.0 & $47.70887-0.05512 . \mathrm{T}$ & ** & 80.4 \\
\hline ANFC 9 & $56.33325-0.03488 . T$ & * * & 85.8 & $54.03569-0.03414 . T$ & ** & 82.1 \\
\hline ANFc 5 & $54.02720-0.03545 . T$ & * * & 73.3 & $54.7625-0.0315 . T$ & ** & 76.1 \\
\hline
\end{tabular}


Carioca Aruã, TAA Dama, and Branquinho showed the smallest changes in the $\mathrm{L}$ value during the seed storage period evaluated.

\section{Correlation between the traits evaluated}

The analyses of correlation between the traits evaluated in the two water treatments are shown in Figures 1 and 2. Under irrigation, a positive and significant correlation $(p$ $\leq 0.05)$ was observed between LT and NDF $(r=0.4)$, LA and NN ( $\mathrm{r}=0.4)$, LA and RCD $(\mathrm{r}=0.5)$, LA and TDM $(\mathrm{r}=0.7), \mathrm{NN}$ and $\mathrm{PH}(\mathrm{r}=0.5), \mathrm{NN}$ and $\mathrm{RCD}(\mathrm{r}=0.3)$, $\mathrm{NN}$ and TDM $(\mathrm{r}=0.4), \mathrm{PH}$ and NDM $(\mathrm{r}=0.5), \mathrm{RCD}$ and TDM $(r=0.5)$, NSP and NPP $(r=0.5)$, NSP and NEL (0.4), NSP and SY ( $\mathrm{r}=0.7)$, NSP and CT ( $\mathrm{r}=0.5)$, NPP and NEL $(r=0.6)$, NEL and CT $(r=0.4)$, and SY and CT $(\mathrm{r}=0.3)$. In contrast, there was a negative and significant correlation $(p \leq 0.05)$ between RWC and LT ( $\mathrm{r}=-0.5)$, LT and $\mathrm{PH}(\mathrm{r}=-0.4)$, LT and SY $(\mathrm{r}=-0.3)$, NN and NUP $(\mathrm{r}$ $=-0.4), \operatorname{RCD}$ and NUP $(\mathrm{r}=-0.3)$, TDM and NPP $(\mathrm{r}=$ $-0.3)$, and TDM and NUP ( $\mathrm{r}=-0.4)$ (Figure 1).

Under water deficit, there were positive and significant correlations $(p \leq 0.05)$ for LT and LA $(\mathrm{r}=$ $0.4)$, LT and PH $(r=0.4)$, LT and TDM $(r=0.5)$, LA and $\mathrm{NN}(\mathrm{r}=0.4)$, LA and PH $(\mathrm{r}=0.3)$, LA and TDM $(\mathrm{r}=$ $0.8)$, LA and CT ( $\mathrm{r}=0.5), \mathrm{NN}$ and $\mathrm{PH}(\mathrm{r}=0.5), \mathrm{NN}$ and $\operatorname{TDM}(\mathrm{r}=0.4), \mathrm{PH}$ and TDM $(\mathrm{r}=0.6), \mathrm{RCD}$ and NDM $(\mathrm{r}=0.3)$, TDM and NDM $(\mathrm{r}=0.3)$, TDM and CT $(\mathrm{r}=$

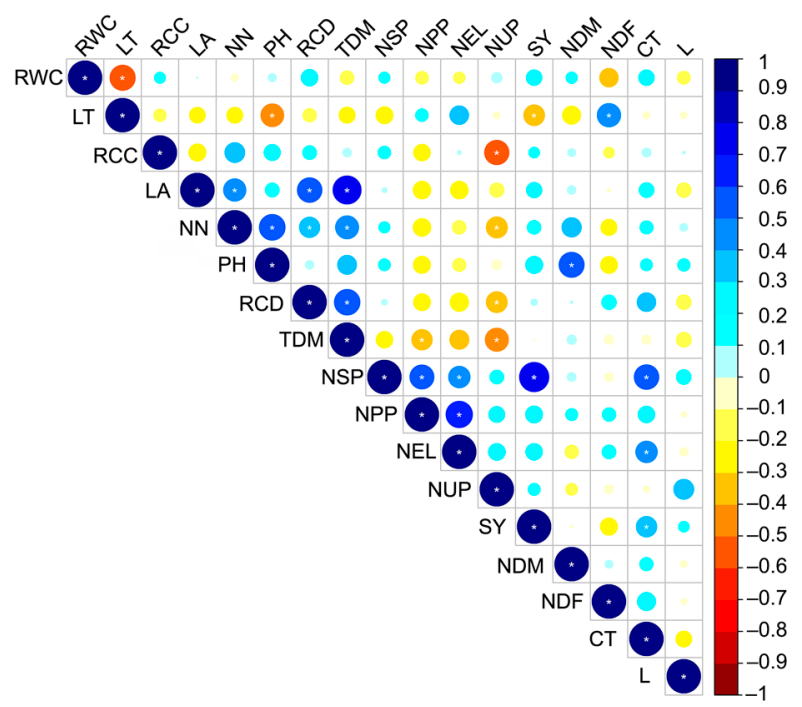

Figure 1 - Analysis of phenotypic correlation between the traits evaluated in 30 common bean genotypes in the irrigated treatment. Relative water content (RWC), leaf temperature (LT), relative chlorophyll content (RCC), leaf area (LA), number of nodes (NN), plant height (PH), root collar diameter (RCD), total dry matter (TDM), number of seeds per plant (NSP), number of pods per plant (NPP), number of empty locules (NEL), number of unviable pods (NUP), seed yield (SY), number of days to maturity (NDM), number of days to flowering (NDF), cooking time (CT), and value of $\mathrm{L}$ (lightness). ${ }^{*}$ Significant at $5 \%$ probability by the $t$ test.
$0.3), \mathrm{NSP}$ and NPP $(\mathrm{r}=0.5), \mathrm{NSP}$ and SY (0.7), NSP and L (0.3), and NPP and SY (0.6). In contrast, RWC and LA $(\mathrm{r}=-0.5)$, RWC and TDM $(\mathrm{r}=-0.4)$, NUP and NDM $(\mathrm{r}$ $=-0.4)$, and SY and NDM $(r=-0.3)$ exhibited negative and significant correlations $(p \leq 0.05)$ (Figure 2).

The results of the correlation analyses, regardless of the water regime adopted, showed no significant correlation between the $\mathrm{L}$ value and cooking time (Figures 1 and 2). The correlation analyses of the $\mathrm{L}$ value between the storage times evaluated showed that after 30 days of storage, significant positive correlations occurred between the storage times, regardless of the water regime adopted (Table 4).

\section{Discussion}

Response to drought is a complex characteristic regulated by various genes and depends on the genotype, period, and severity of water deficit (Ramirez-Vallejo and Kelly, 1998). In common bean, drought affects both shoot growth and root growth, reducing cell expansion, stomatal conductance, photosynthetic and respiration rates, biomass accumulation, and nutrient uptake. At flowering, drought leads to abortion of flowers and pods, directly affecting seed yield (Asfaw and Blair, 2012).

The $\mathrm{G} \times \mathrm{WT}$ interaction $(p \leq 0.05)$ had a significant effect on the traits NPP, NEL, SY, and CT, indicating that some genotypes showed differential performance

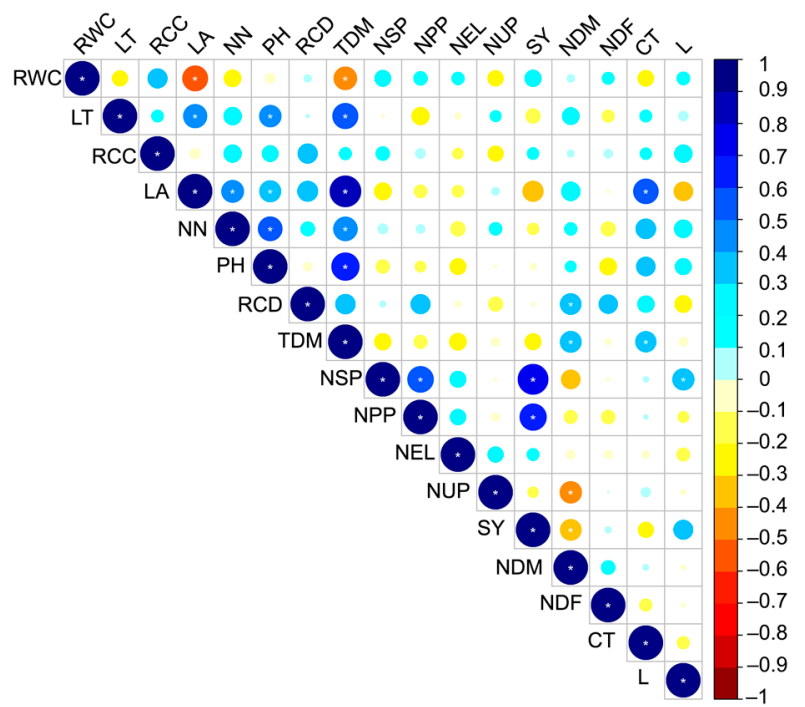

Figure 2 - Analysis of phenotypic correlation between the traits evaluated in 30 common bean genotypes under water deficit. Relative water content (RWC), leaf temperature (LT), relative chlorophyll content (RCC), leaf area (LA), number of nodes (NN), plant height $(\mathrm{PH})$, root collar diameter (RCD), total dry matter (TDM), number of seeds per plant (NSP), number of pods per plant (NPP), number of empty locules (NEL), number of unviable pods (NUP), seed yield (SY), number of days to maturity (NDM), number of days to flowering (NDF), cooking time (CT), and value of $\mathrm{L}$ (lightness). * Significant at $5 \%$ probability by the $t$ test. 
Table 4 - Analysis of correlation of the $L$ value between 10 storage times under irrigated and water deficit treatment.

\begin{tabular}{|c|c|c|c|c|c|c|c|c|c|c|}
\hline \multicolumn{11}{|c|}{ Irrigated } \\
\hline Storage time & 0 & 30 & 60 & 90 & 120 & 150 & 180 & 210 & 240 & 270 \\
\hline \multicolumn{11}{|l|}{ days } \\
\hline 0 & 1.0 & $0.8^{*}$ & $0.7^{*}$ & $0.6^{*}$ & $0.6^{*}$ & $0.6^{*}$ & $0.6^{*}$ & $0.6^{*}$ & $0.6^{*}$ & $0.6^{*}$ \\
\hline 30 & & 1.0 & $0.9^{*}$ & $0.9^{*}$ & $0.9^{*}$ & $0.9^{*}$ & $0.9^{*}$ & $0.9^{*}$ & $0.9^{*}$ & $0.9^{*}$ \\
\hline 60 & & & 1.0 & $0.9^{*}$ & $0.9^{*}$ & $0.9^{*}$ & $0.9^{*}$ & $0.9^{*}$ & $0.9^{*}$ & $0.9^{*}$ \\
\hline 90 & & & & 1.0 & $0.9^{*}$ & $0.9^{*}$ & $0.9^{*}$ & $0.9^{*}$ & $0.9^{*}$ & $0.9^{*}$ \\
\hline 120 & & & & & 1.0 & $0.9^{*}$ & $0.9^{*}$ & $0.9^{*}$ & $0.9^{*}$ & $0.9^{*}$ \\
\hline 150 & & & & & & 1.0 & $1.0^{*}$ & $1.0^{*}$ & $1.0^{*}$ & $1.0^{*}$ \\
\hline 180 & & & & & & & 1.0 & $1.0^{*}$ & $1.0^{*}$ & $1.0^{*}$ \\
\hline 210 & & & & & & & & 1.0 & $1.0^{*}$ & $1.0^{*}$ \\
\hline 240 & & & & & & & & & 1.0 & $1.0^{*}$ \\
\hline 270 & & & & & & & & & & 1.0 \\
\hline \multicolumn{11}{|c|}{ Deficit } \\
\hline Storage time & 0 & 30 & 60 & 90 & 120 & 150 & 180 & 210 & 240 & 270 \\
\hline \multicolumn{11}{|l|}{ days } \\
\hline 0 & 1.0 & $0.8^{*}$ & $0.7^{*}$ & $0.7^{*}$ & $0.7^{*}$ & $0.6^{*}$ & $0.6^{*}$ & $0.6^{*}$ & $0.6^{*}$ & $0.6^{*}$ \\
\hline 30 & & 1.0 & $0.9^{*}$ & $0.9^{*}$ & $0.9^{*}$ & $0.9^{*}$ & $0.9^{*}$ & $0.9^{*}$ & $0.9^{*}$ & $0.9^{*}$ \\
\hline 60 & & & 1.0 & $0.9^{*}$ & $0.9^{*}$ & $0.9^{*}$ & $0.9^{*}$ & $0.9^{*}$ & $0.9^{*}$ & $0.9^{*}$ \\
\hline 90 & & & & 1.0 & $0.9^{*}$ & $0.9^{*}$ & $0.9^{*}$ & $0.9^{*}$ & $0.9^{*}$ & $0.9^{*}$ \\
\hline 120 & & & & & 1.0 & $0.9^{*}$ & $0.9^{*}$ & $0.9^{*}$ & $0.9^{*}$ & $0.9^{*}$ \\
\hline 150 & & & & & & 1.0 & $1.0^{*}$ & $0.9^{*}$ & $0.9^{*}$ & $1.0^{*}$ \\
\hline 180 & & & & & & & 1.0 & $1.0^{*}$ & $0.9^{*}$ & $0.9^{*}$ \\
\hline 210 & & & & & & & & 1.0 & $0.9^{*}$ & $0.9^{*}$ \\
\hline 240 & & & & & & & & & 1.0 & $1.0^{*}$ \\
\hline 270 & & & & & & & & & & 1.0 \\
\hline
\end{tabular}

according to the water treatment. Variation in CVs obtained from $4.6 \%$ for NDM to $36.9 \%$ for $\mathrm{PH}$ are in accordance to reports in the literature on the response to water deficit in common bean (Gonçalves et al., 2015; Gonçalves et al., 2019; Ribeiro et al., 2019a; Arruda et al., 2019; Sánchez-Reinoso et al., 2020).

In this study, the stress intensity index was $45.4 \%$, which led to a significant reduction in RWC, LA, PH, TDM, NSP, NPP, SY, and NDM compared to the irrigated treatment. Water deficit compromises flowering and pod formation in common bean, interfering in production components and seed yield. The number of pods per plant was the production component most affected by water deficit (Barrios et al., 2005). In contrast, leaf temperature and in cooking time increased in genotypes under water deficit.

The RWC can be a parameter of the water status of the plants; thus, the significant reduction of $19.5 \%$ confirms the effectiveness of the water deficit applied. Under water deficit, one of the first plant reactions is stomatal closure to prevent water loss through transpiration. The reduction of the transpiration rate increases leaf temperature, which may lead to a reduction in photosynthetic rates. In addition, stomatal closure reduces the $\mathrm{CO}_{2}$ inflow in the leaves, negatively affecting photosynthesis and consequently reducing seed yield (Duan et al., 2018; Tardieu et al., 2018).
The early yellowing of leaves is one of the most expressive visual symptoms of plants subjected to water deficit and the relative chlorophyll content allows evaluating this parameter. In this study, water restriction led to this change in leaf color; nevertheless, it did not differentiate statistically genotypes or water treatments $(p>0.05)$. Gonçalves et al. (2019) evaluated 30 common bean genotypes regarding drought tolerance and did not observe an effect of the water regime on the relative chlorophyll content. The authors did not use this trait in the selection of drought tolerant genotypes. However, the authors report that the relative chlorophyll content is affected by drought and that the large number of genotypes evaluated may have contributed to the absence of significant statistical differences.

Reduction in leaf area caused by water deficit may be a strategy adopted by plants to reduce water loss through transpiration; however, it directly affects the photosynthetic rate and may interfere in seed production (Arve et al., 2011). Smith et al. (2019) evaluated the response of common bean genotypes regarding tolerance to water deficit and observed reduction in the leaf area of plants under stress. Water deficit also reduced $\mathrm{PH}$, which can be explained by the limitation of cell expansion caused by water deficit, directly affecting plant growth and seed yield, according to Ribeiro et al. (2019b), corroborating our results.

In addition, TDM declined by $44.0 \%$ in the intermittent water deficit. This reduction decreases the photosynthetic rate, increasing plant growth inhibitors and reducing hormone produciton, especially auxins and cytokinins (Hayat and Ahmad, 2007). Thus, the following genotypes were significant to keep their TDM under water deficit: IAC Carioca Eté, IAC Carioca Tybatã, IAC Imperador, IAC 1850, IAC 1849 Polaco, Gen TS 4-8, BRS Horizonte, BRS Requinte, FC 402, BRSMG Madrepérola, IPR Quero-Quero, IPR Campos Gerais, IPR Curió, TAA Dama, and TAA Gol (Table 1).

The water treatment negatively affected production components (NSP and NPP); however, cultivar IAC 1850 had the best performance for both these traits and it is responsive to irrigation, in absolute values (Table 2). Asfaw and Blair (2014) also observed reduction in the number of seeds per pod and number of pods per common bean plant under water deficit, most severely affecting yield in some genotypes evaluated, due to existing variability.

Seed yield declined because of water deficit, in agreement with Androcioli et al. (2020), Dipp et al. (2017), and Gonçalves et al. (2019). Ideal genotypes maintain their production levels even under water deficit conditions. Therefore, cultivars IAC 1850, TAA Dama, and IAC Carioca Aruã are expressive for exhibiting the highest seed yields under water restriction in absolute values.

The higher drought tolerance index, the higher the seed yield in both irrigated and water deficit conditions 
compared to the mean production of all cultivars under water deficit conditions (Darkwa et al., 2016; Dipp et al., 2017). Based on the DTI, the IAC 1850, TAA Dama, IAC Carioca Aruã, and BRMG Madrepérola genotypes were more tolerant to water deficit.

Beebe et al. (2013) affirm that one of the main mechanisms that plants use to withstand water deficit is rapid phenological development, with a smaller number of days to flowering and maturity. However, the authors reported that water deficit did not change the NDF of the genotypes evaluated, in agreement with ChavesBarrantes et al. (2018).

The mean cooking time of the genotypes evaluated increased by $38.5 \%$ under water deficit, corroborating Kazai et al. (2019). According to Selmar and Kleinwächter (2013), plants under water deficit accumulate high concentrations of secondary metabolites, especially simple and complex phenols. The higher contents of lignin and polyphenols in cotyledons limit the ability of water imbibition of seeds, making them harder and therefore requiring longer cooking time (Esteves et al., 2002).

In addition to yield aspects, post-harvest quality of the seed is a decisive factor for adoption of a cultivar, especially tolerance to seed coat darkening during seed storage. The analysis of variance of the $\mathrm{L}$ value showed significant effects $(p \leq 0.01)$ for genotypes, storage time, and interactions $\mathrm{G} \times \mathrm{WR}, \mathrm{G} \times \mathrm{ST}, \mathrm{WR} \times \mathrm{ST}$, and $\mathrm{G} \times$ WR $\times$ ST. The existence of genetic variability for the $\mathrm{L}$ value allows the selection of carioca common bean genotypes more tolerant to seed coat darkening during storage, with greater acceptance by consumers (Arns et al., 2018). There were no significant differences $(p$ $>0.05$ ) for the $\mathrm{L}$ value between the water treatments, indicating that water deficit did not affect seed coat darkening of common bean, corroborating Silva et al. (2020).

The common bean genotypes evaluated regarding seed coat darkening after harvest can be classified into genotypes of slow darkening or regular darkening (Elsadr et al., 2011). Regardless of the water regime, regression equations show that all the genotypes darkened during the storage time periods evaluated. However, cultivars IAC 1849 Polaco, ANFc 5, ANFc 9, BRSMG Madrepérola, IAC Carioca Aruã, TAA Dama, and Branquinho had the least negative values of the regression equation angular coefficient for the $\mathrm{L}$ value. This means that the respective genotypes showed less darkening of seed coat during 270 days of storage and are thus classified as genotypes of slow darkening, increasing their shelf life.

In contrast, genotypes with regular darkening of the seed coat should be marketed soon after harvest, since darkening during storage lowers their quality and market value, hindering their sale (Arns et al., 2018), since consumers associate dark beans to older beans and longer cooking time (Silva et al., 2008). Silva et al. (2014), Siqueira et al. (2014), and Spitti et al. (2019) evaluated beans from common bean cultivars over different storage periods and found different responses at the genotype level, highlighting the genetic variability for this trait.

For carioca seed coat beans, Ribeiro et al. (2008) proposed that the value of $\mathrm{L} \geq 55.0$ as ideal for the light hue of the seed. However, after 270 days of storage, no genotype evaluated achieved this standard value.

The correlation analysis estimates the direction and magnitude of relationship between two traits, allowing evaluation of indirect responses in traits of low heritability or problems of identification and measurement (Cruz et al., 2014). The correlation analyses showed that NSP and NPP had significant positive values of high magnitude with SY under water deficit (Figure 2). Thus, the selection of higher yielding genotypes under water restriction can be performed indirectly based on NSP and NPP. These results are in agreement with Kazai et al. (2019), who also observed positive and significant correlation between NPP and SY and between NSP and SY.

One significant trait for growers' acceptance regarding a common bean cultivar is the speed of seed coat darkening (Silva et al., 2008). Thus, it is essential to investigate the association between seed coat darkening and cooking time. The correlation analyses did not show significant correlation between the $\mathrm{L}$ value and cooking time, regardless of the water regime adopted (Figures 1 and 2). Thus, beans from cultivars with the regular seed coat darkening trait do not necessarily have longer cooking time compared to beans of cultivars with the slow seed coat darkening trait.

Another important question regards the suitable storage period for the analyses of bean seed coat darkening. A storage period longer than 90 days may lead to delay in breeding programs, hindering to grow three generations a year in the case of common bean (Silva et al., 2008).

After 30 days of storage, regardless of the water regime, significant positive correlations were observed between the storage times evaluated (Table 4). Thus, the selection of common bean genotypes tolerant to seed coat darkening could be carried out effectively at 30 days of storage.

Among all the genotypes evaluated, cultivar IAC 1850 had the best yield under water deficit and the highest DTI value, although it did not have a stable response. Nevertheless, for better acceptance of a new cultivar by growers and the market, aspects related to the technological quality of bean seeds should also be considered, such as cooking time and seed coat color, especially tolerance to seed coat darkening. Genotypes IAC 1849 Polaco, ANFc 5, ANFc 9, BRSMG Madrepérola, IAC Carioca Aruã, TAA Dama, and Branquinho are significant because they do not differ statistically in seed yield under water deficit from cultivar IAC 1850 and kept a lighter hue in seed coats during the storage periods. 


\section{Acknowledgments}

The authors thank the São Paulo Research Foundation (FAPESP) for funding the research, and the Coordination for the Improvement of Higher Level Personnel (CAPES), for the granting of the scholarships, which enabled carrying out this study. The first author thanks CAPES for the doctoral scholarship.

\section{Authors' Contributions}

Conceptualization: Gonçalves, J.G.R.; Chiorato, A.F.; Carbonell, S.A.M. Data acquisition: Paulino, J.F.C.; Almeida, C.P.; Gonçalves, J.G.R. Data analysis: Gonçalves, G.M.C. Writing and editing: Gonçalves, G.M.C.; Gonçalves, J.G.R.; Chiorato, A.F.; Carbonell, S.A.M.

\section{References}

Androcioli, L.G.; Zeffa, D.M.; Alves, D.S.; Tomaz, J.P.; ModaCirino, V. 2020. Effect of water deficit on morphoagronomic and physiological traits of common bean genotypes with contrasting drought tolerance. Water 12: 1-13.

Arns, F.D.; Ribeiro, N.D.; Mezzomo, H.C.; Steckling, S.D.M.; Kläsener, G.R.; Casagrande, C.R. 2018. Combined selection in carioca beans for grain size, slow darkening and fastcooking after storage times. Euphytica 214: 1-12.

Arruda, I.M.; Moda-Cirino, V.; Koltun, A.; Santos, O.J.A.P.; Moreira, R.S.; Moreira, A.F.P.; Gonçalves, L.S.A. 2018. Physiological, biochemical and morphoagronomic characterization of drought-tolerant and drought-sensitive bean genotypes under water stress. Physiology and Molecular Biology of Plants 24: 1059-1067.

Arruda, I.M.; Moda-Cirino, V.; Koltun, A.; Zeffa, D.M.; Nagashima, G.T.; Gonçalves, L.S.A. 2019. Combining Ability for Agromorphological and Physiological Traits in Different Gene Pools of Common Bean Subjected to Water Deficit. Agronomy 9: 1-13.

Arve, L.; Torres, S.; Olsen, J.; Tanino, K.K. 2011. Stomatal responses to drought stress and air humidity. p. 267-280. In: Shanker, A.K.; Venkateswarlu, B., eds. Abiotic stress in plants - mechanisms and adaptations. InTech, Rijeka, Croatia.

Asfaw A.; Blair, M.W. 2012. Quantitative trait loci for rooting pattern traits of common beans grown under drought stress versus nonstress conditions. Molecular Breeding 30: 681695.

Asfaw, A.; Blair, M.W. 2014. Quantification of drought tolerance in Ethiopian common bean varieties. Agricultural Sciences 5: 124-139.

Barili, L.D.; Vale, N.M.; Moura, L.M.; Paula, R.G.; Silva, F.F.; Carneiro, J.E.S. 2016. Genetic progress resulting from fortythree years of breeding of the carioca common bean in Brazil. Genetics and Molecular Research 15: 1-11.

Barrios, A.N.; Hoogenboom, G.; Nesmith, D.S. 2005. Drought stress and the distribution of vegetative and reproductive traits of a bean cultivar. Scientia Agricola 62: 18-22.
Beebe, S.E.; Rao, I.M.; Blair, M.W.; Acosta-Gallegos, J.A. 2013. Phenotyping common beans for adaptation to drought. Frontiers in Physiology 4: 1-20.

Chaves-Barrantes, N.F.; Polania, J.A.; Muñoz-Perea, C.G.; Rao, I.M.; Beebe, S.E. 2018. Phenotypic characterization of common bean germplasm for resistance to terminal drought $=$ Caracterizacion fenotípica por resistência a sequía terminal de germoplasma de frijol comum. Agronomía Mesoamericana 29: 1-17 (in Spanish, with abstract in English).

Chiorato, A.F.; Carbonell, S.A.M.; Vencovsky, R.; Fonseca Júnior, N.S.; Pinheiro, J.B. 2010. Genetic gain in the breeding program of common beans at IAC from 1989 to 2007. Crop Breeding and Applied Biotechnology 10: 329-336.

Cruz, C.D. 2013. GENES: a software package for analysis in experimental statistics and quantitative genetics. Acta Scientiarum 35: 271-276.

Cruz, C.D.; Regazzi, A.J.; Carneiro, P.C.S. 2014. Biometric Models Applied to Genetic Improvement $=$ Modelos Biométricos Aplicados ao Melhoramento Genético. Editora UFV, Viçosa, MG, Brazil (in Portuguese).

Darkwa, K.; Ambachew, D.; Mohammed, H.; Asfaw, A.; Blair, M.W. 2016. Evaluation of common bean (Phaseolus vulgaris L.) genotypes for drought stress adaptation in Ethiopia. The Crop Journal 4: 367-376.

Dipp, C.C.; Marchese, J.A.; Woyann, L.G.; Bosse, M.A.; Roman, M.H.; Gobatto, D.R.; Paludo, F.; Fedrigo, K.; Kovali, K.K.; Finatto, T. 2017. Drought stress tolerance in common bean: what about highly cultivated Brazilian genotypes? Euphytica 213: 1-16.

Duan, H.; Chaszar, B.; Lewis, J.D.; Smith, R.A.; Huxman, T.E.; Tissue, D.T. 2018. $\mathrm{CO}_{2}$ and temperature effects on morphological and physiological traits affecting risk of drought-induced mortality. Tree Physiology 38: 1138-1151.

Egu, A.M. 2016. Evaluation of morphological aspects of common bean (Phaseolus vulgaris L.) genotypes for post-flowering drought resistance in Rift Valley of Ethiopia. African Journal of Agricultural Research 11: 3020-3026.

Elsadr, H.T.; Wright, L.; Pauls, K.P.; Bett, K.E. 2011. Characterization of seed coat post harvest darkening in common bean (Phaseolus vulgaris L.). Theoretical and Applied Genetics 123: $1467-1472$.

Esteves, A.M.; Abreu, C.M.P.; Santos, C.D.; Corrêa, A.D. 2002. Chemical and enzymatic comparison of six bean lineages (Phaseolus Vulgaris L.). Ciência e Agrotecnologia 26: 999-1005 (in Portuguese, with abstract in English).

Fahad, S.; Bajwa, A.A.; Nazir, U.; Anjum, S.A.; Farooq, A.; Zohaib, A.; Sadia, S.; Nasim, W.; Adkins, S.; Saud, S.; Ihsan, M.Z.; Alharby, H.; Wu, C.; Wang, D.; Huang, J. 2017. Crop production under drought and heat stress: plant responses and management options. Frontiers in Plant Science 8: 1-16.

Faria, L.C.; Peloso, M.J.D.; Melo, L.C.; Costa, J.G.C.; Rava, C.A.; Díaz, J.L.C.; Faria, J.C.; Silva, H.T.; Sartorato, A.; Bassinello, P.Z.; Trovo, J.B.F. 2008. BRS Cometa: a carioca common bean cultivar with erect growth habit. Crop Breeding and Applied Biotechnology 8: 167-169.

Fischer, R.A.; Maurer, R. 1978. Drought resistance in spring wheat cultivars. I. Grain yield responses. Australian Journal of Agricultural Research 29: 897-912. 
Gonçalves, J.G.R.; Andrade, E.R.; Silva, D.A.; Esteves, J.A.F.; Chiorato, A.F. Carbonell, S.A.M. 2019. Drought tolerance evaluated in common bean genotypes. Ciência e Agrotecnologia 43: 1-9.

Gonçalves, J.G.R.; Chiorato, A.F.; Silva, D.A.; Esteves, J.A.F.; Bosetti, F.; Carbonell, S.A.M. 2015. Combining ability in common bean cultivars under drought stress. Bragantia 74: 149-155.

Hayat, S.; Ahmad, A. 2007. Salicylic Acid: A Plant Hormone. Springer, Dordrecht, The Netherlands.

Jamaux, I.; Steinmetz, A.; Belhassen, E. 1997. Looking for molecular and physiological markers of osmotic adjustment in sunflower. New Phytologist 137: 117-127.

Kazai, P.; Noulas, C.; Khah, E.; Vlachostergios, D. 2019. Yield and seed quality parameters of common bean cultivars grown under water and heat stress field conditions. AIMS Agriculture and Food 4: 285-302.

Lanna, A.C.; Mitsuzono, S.T.; Terra, T.G.R.; Vianello, R.P.; Carvalho, M.A.F. 2016. Physiological characterization of common bean (Phaseolus vulgaris L.) genotypes, water-stress induced with contrasting response towards drought. Australian Journal of Crop Science 10: 1-6.

Mutava, R.N.; Prince, S.J.K.; Syed, N.H.; Song, L.; Valliyodan, B.; Chen, W.; Nguyen, H.T. 2015. Understanding abiotic stress tolerance mechanisms in soybean: a comparative evaluation of soybean response to drought and flooding stress. Plant Physiology and Biochemistry 86: 109-120.

Oya, T.; Nepomucemo, A.L.; Neumaier, N.; Farias, J.R.B.; Tobita, S.; Ito, O. 2004. Drought tolerance characteristics of Brazilian soybean cultivars: evaluation and characterization of drought tolerance of various Brazilian soybean cultivars in the field. Plant Production Science 7: 129-137.

Proctor, J.R.; Watts, B.M. 1987. Development of a modified Mattson bean cooker procedure based on sensory panel cookability evaluation. Canadian Institute of Food Science and Technology Journal 20: 9-14.

Ramirez-Vallejo, P.; Kelly, J.D. 1998. Traits related to drought resistance in common bean. Euphytica 99: 127-136.

Rao, I.M. 2014. Advances in improving adaptation of common bean and Brachiaria forage grasses to abiotic stresses in the tropics. p. 847-889. In: Pessarakli, M., ed. Handbook of plant and crop physiology. CRC Press, Boca Raton, FL, USA.

Ribeiro, N.D.; Casagrande, C.R.; Mezzomo, H.C.; Kläsener, G.R.; Steckling, S.M. 2019a. Consumer preference and the technological, cooking and nutritional quality of carioca beans. Semina: Ciências Agrárias 40: 651-664.

Ribeiro, N.D.; Storck, L.; Poersch, N.L. 2008. Classify the common bean commerciais lots by the clarity of the grain tegument. Ciência Rural 8: 2042-2045 (in Portuguese, with abstract in English).
Ribeiro, T.; Silva, D.A.; Esteves, J.A.F.; Azevedo, C.V.G.; Gonçalves, J.G.R.; Carbonell, S.A.M.; Chiorato, A.F. 2019 b. Evaluation of common bean genotypes for drought tolerance. Bragantia 78: 1-11.

Sánchez-Reinoso, A.D.; Ligarreto-Moreno, G.A.; Restrepo-Díaz, H. 2020. Evaluation of drought indices to identify tolerant genotypes in common bean bush. Journal of Integrative Agriculture 19: 99-107.

Selmar, D.; Kleinwächter, M. 2013. Influencing the product quality by deliberately applying drought stress during the cultivation of medicinal plants. Industrial Crops and Products 42: 558-566.

Silva, A.N.; Ramos, M.L.G.; Ribeiro Júnior, W.Q.; Alencar, E.R.; Silva, P.C.; Lima, C.A.; Vinson, C.C.; Silva, M.A.V. 2020. Water stress alters physical and chemical quality in grains of common bean, triticale and wheat. Agricultural Water Management 231: $1-10$

Silva, F.C.; Melo, P.G.S.; Pereira, H.S.; Melo, L.C. 2014. Genetic control and estimation of genetic parameters for seed-coat darkening of carioca beans. Genetic and Molecular Research 13: 6486-6496.

Silva, G.S.; Ramalho, M.A.O.; Abreu, A.F.B.; Silva, F.B. 2008. Genetic control of early grain darkening of carioca common bean. Crop Breeding and Applied Biotechnology 8: 299-304.

Siqueira, B.; Pereira, W.J.; Batista, K.A.; Oomah, B.D.; Fernandes, K.F.; Bassinello, P.Z. 2014. Influence of storage on darkening and hardening of slow- and regular-darkening carioca bean (Phaseolus vulgaris L.) genotypes. Journal of Agricultural Studies 2: 87-104.

Smith, M.R.; Veneklaas, E.; Polania, J.; Rao, I.M.; Beebe, S.E.; Merchant, A. 2019. Field drought conditions impact yield but not nutritional quality of the seed in common bean (Phaseolus vulgaris L.). PLoS One 14: e0217099.

Spitti, A.M.D.S.; Carbonell, S.A.M.; Dias, C.T.S.; Sabino, L.G.; Carvalho, C.R.L.; Chiorato, A.F. 2019. Carioca bean genotypes for tolerance to grain darkening by natural and accelerated methods. Ciência e Agrotecnologia 43: 1-10.

Tardieu, F.; Simonneau T.; Muller, B. 2018. The physiological basis of drought tolerance in crop plants: a scenario-dependent probabilistic approach. Annual Review of Plant Biology 69: 733-759.

Zeffa, D.M.; Moda-Cirino, V.; Medeiros, I.A.; Freiria, G.H.; Neto, J.S.; Ivamoto-Suzuki, S.T.; Delfini, J.; Scapim, C.A.; Gonçalves, L.S.A. 2020. Genetic progress of seed yield and nitrogen use efficiency of Brazilian carioca common bean cultivars using bayesian approaches. Frontiers in Plant Science 11: 1-14. 